\title{
An African American man with dysphagia: a unique initial presentation of sarcoidosis
}

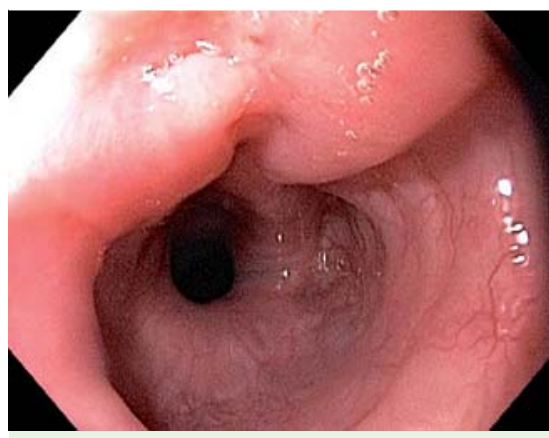

Fig. 1 Endoscopic image of mid-esophageal lesion with a focal area of depression.

A 46-year-old African American man whose past medical history was only notable for hypertension was referred to the gastrointestinal clinic with a 1-year history of dysphagia. The dysphagia related only to solid foods and had been progressively worsening over the preceding few months. The patient described the sensation of food getting stuck at the level of the mid to lower chest. He denied any odynophagia or difficulty with swallowing liquids. He denied any chronic heartburn symptoms, regurgitation, hematemesis, or weight loss. Physical examination was unremarkable. Laboratory studies including complete blood count, basic metabolic panel, and liver function tests were within normal limits. A barium swallow was performed and revealed a mid-esophageal stricture with obstruction of approximately $50 \%$ of the lumen.

On esophagogastroduodenoscopy, there was a nearly circumferential mid-esophageal lesion noted from 28 to $33 \mathrm{~cm}$ from the incisors ( $\bullet$ Fig. 1). The lesion's overlying mucosa appeared normal, with no evidence of ulceration or inflammation. There was a focal area of depression in the lesion resembling the beginning of a diverticulum. The lesion caused narrowing of the mid-esophageal lumen, and the standard upper endoscope was able to traverse the area with mild resistance. The stomach and duodenum were unremarkable. Cold forceps biopsies were obtained from the esophageal lesion and revealed granulomatous inflammation. Acid-fast bacilli staining of the tissue was negative.

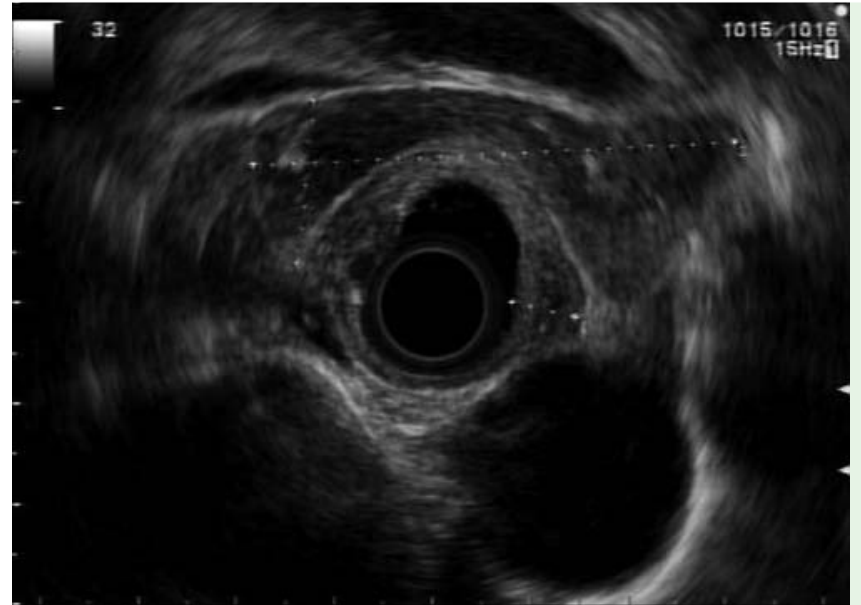

Fig. 2 Endoscopic ultrasound (EUS) image of the esophageal lesion with thickened mucosal and submucosal layers and mediastinal lymphadenopathy.

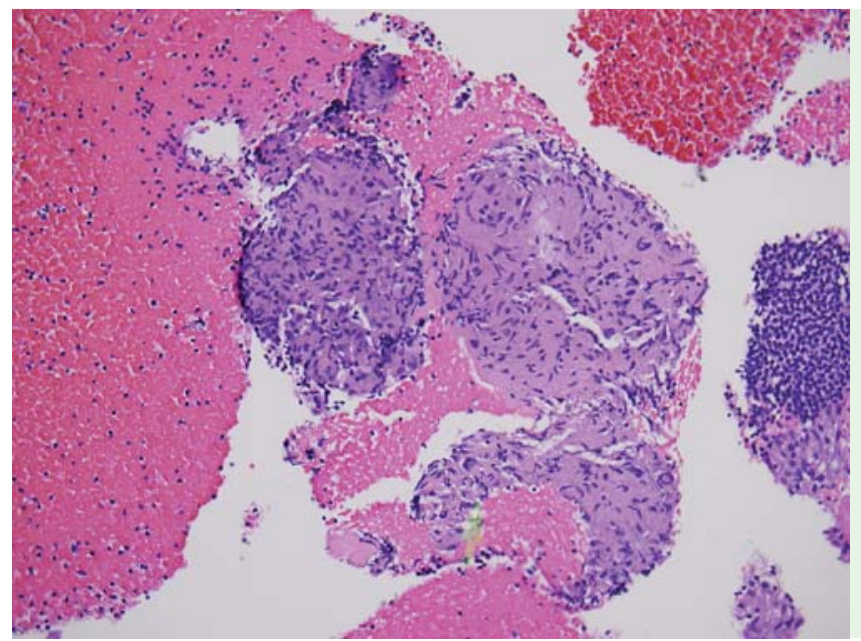

Fig. 3 Histological appearance of lymph node sample (hematoxylin and eosin stain, $\times 100$ ) showing cellular changes consistent with granulomatous inflammation.

In light of the biopsy results, a computed tomography (CT) scan of the chest was obtained. In addition to thickening of the mid esophagus, CT demonstrated significant mediastinal and right hilar lymphadenopathy. Endoscopic ultrasound (EUS) was employed to further evaluate the esophagus and to obtain a biopsy of the mediastinal lymphadenopathy. On EUS the esophageal lesion was visualized as thickened mucosal and submucosal layers ( Fig.2). At $32 \mathrm{~cm}$ from the incisors, the esophageal lesion was almost circumferential and causing narrowing. The mucosa at $32 \mathrm{~cm}$ was measured as $6.7 \mathrm{~mm}$. Several enlarged lymph nodes were seen in the mediastinum at the level of 32,25 , and $21 \mathrm{~cm}$ from the incisors. Fine-needle aspiration (FNA) without suction of the lymph node at $32 \mathrm{~cm}$ was performed with a 22-gauge needle, and cell block preparation revealed cellular changes consistent with granulomatous inflammation (๑ Fig.3).

The diagnosis of sarcoidosis requires a compatible clinical picture, histology of noncaseating granulomas, and exclusion of other granulomatous diseases. EUSguided FNA has been shown to be a safe and sensitive method to detect granulomas in the lymph nodes of patients suspected of sarcoidosis [1]. In the light of this patient's CT scan findings and evidence of granulomas in the mediastinal lymph nodes and the esophagus, a diagnosis of mediastinal sarcoidosis with esophageal involvement was made. 
Esophageal involvement in sarcoidosis is rare. Dysphagia is the most common presentation and can be attributed to dysmotility from neuropathy or myopathy. It may also be due to mechanical obstruction from infiltration of the esophageal wall or extrinsic compression from subcarinal lympadenopathy. As in our case, granulomatous infiltration of the esophageal wall can produce a thickened, distorted, and narrow esophagus. Infiltration of the wall, producing aphthous lesions, plaque-like protrusions, and esophageal strictures has also been reported in the literature [2].

Given the low frequency of sarcoidosis affecting the esophagus, there are no trials comparing various treatment modalities. The role of steroids in the treatment of gastrointestinal sarcoidosis is unclear, although case reports indicate a potential benefit. Esophageal inflammation and gastric ulceration related to infiltration from sarcoidosis have been shown to respond to antisecretory therapy $[2,3]$.

\section{Acknowledgements}

The authors thank Mark Wu, MD, and Suvarna Deshmukh, MD, of the Department of Pathology, University of California - Irvine, USA.

Endoscopy_UCTN_Code_CCL_1AB_2AC_3AH

Competing interests: None

\section{J. B. Samarasena', E. Chu' ${ }^{2}$, V. R. Muthusamy ${ }^{1}$}

${ }^{1}$ Division of Gastroenterology, University of California - Irvine, Orange, California, USA

${ }^{2}$ Long Beach Gastroenterology Associates, Long Beach, California, USA

\section{References}

1 Bartheld MB, Veselic-Charvat M, Rabe KF et al. Endoscopic ultrasound-guided fine-needle aspiration for the diagnosis of sarcoidosis. Endoscopy 2010; 42: 213-217

2 Ebert EC, Kierson M, Hagspiel KD. Gastrointestinal and hepatic manifestations of sarcoidosis. Am J Gastroenterol 2008; 103: 3184-3192

3 Murdock A, Jacob G. Sarcoidosis of the esophagus presenting macroscopically as Barrett's esophagitis. Am J Gastroenterol 2003; 98: 1661-1662

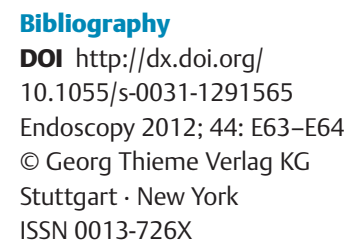

Corresponding author

\section{J. B. Samarasena, MD}

Division of Gastroenterology

University of California - Irvine

Suite 400

333 City Blvd West

Orange

CA 92868

USA

Fax: +1-714-456-7753

jason.samarasena@gmail.com 